\begin{tabular}{l|l} 
REVISTA & $\begin{array}{l}\text { Revista Educación } \\
\text { ISSN: 0379-7082 } \\
\text { ISSN: 2215-2644 } \\
\text { revedu@ gmail.com } \\
\text { Universidad de Costa Rica } \\
\text { Costa Rica }\end{array}$
\end{tabular}

\title{
La retroalimentación escrita en la producción escrita del francés L2
}

Chao Chao, Kuok Wa; Durand, Micheline-Joanne

La retroalimentación escrita en la producción escrita del francés L2

Revista Educación, vol. 45, núm. 2, 2021

Universidad de Costa Rica, Costa Rica

Disponible en: https://www.redalyc.org/articulo.oa?id=44066178004

DOI: https://doi.org/10.15517/revedu.v45i1.43191

\section{(C)}

Esta obra está bajo una Licencia Creative Commons Atribución-NoComercial-SinDerivar 3.0 Internacional. 
Artículos científicos

\title{
La retroalimentación escrita en la producción escrita del francés L2
}

Written Feedback for French L2 Papers

Kuok Wa Chao Chao

Universidad de Costa Rica, Costa Rica

kuok.chao@ucr.ac.cr

DOI: https://doi.org/10.15517/revedu.v45i1.43191 Redalyc: https://www.redalyc.org/articulo.oa?

iD https://orcid.org/0000-0003-0683-1481

\author{
Micheline-Joanne Durand \\ Universidad de Montréal, Canadá \\ mj.durand@umontreal.ca \\ iD https://orcid.org/0000-0002-7540-0186
} $\mathrm{id}=44066178004$

Recepción: 02 Agosto 2020 Aprobación: 29 Septiembre 2020

\section{Resumen:}

Esta investigación tiene como objetivos, por un lado, identificar los tipos de retroalimentación escrita dada por el profesorado a las producciones escritas de sus estudiantes según el modelo de Rodet (2000) y, por otro lado, analizar el uso de la retroalimentación escrita por parte del estudiantado. Es una investigación de tipo colaborativo con tres docentes participantes de francés de la Universidad de Costa Rica y sus grupos de estudiantes. Se emplearon varias técnicas de recolección de datos: grupo de discusión y análisis de las producciones escritas. Se realizó un análisis de contenido por medio de la herramienta QDA Miner. Los principales resultados obtenidos fueron, por parte del personal docente, el uso de la retroalimentación de tipo cognitivo en cuanto al contenido, constructivo en relación con la formulación e informativo con respecto a la intención. El estudiantado encontró una gran utilidad con respecto a la retroalimentación recibida, debido a varias razones como la motivación, la claridad de la información y la indicación de lo que se debía corregir. Por último, las recomendaciones serían utilizar las retroalimentaciones de tipo descriptivas y precisas para que sean útiles para el estudiantado. Tienen que ser también inmediatas. [DM1]

Palabras ClaVE: Retroalimentación escrita, Producción escrita, Educación superior, Francés.

\section{Abstract:}

In order to analyze how students use annotated comments from their instructors, the Rodet model (2000) is used as the basis of this study in order to identify the various types of corrective feedback provided. The subjects included three University of Costa Rica French instructors and their students. Data was gathered from group discussions and student paper analysis with QDA Miner. Results reveal that instructors tend to use cognitive, constructive and informational feedback. Students responded positively to such feedback and stated that it was motivational, delivered clearly and highlighted what needed to be corrected. The author suggests that instructor feedback be more descriptive, specific and immediate to maximize student benefit.

KEYWORDS: Written Feedback, Writing, Higher Education, French.

\section{INTRODUCCIÓN}

La retroalimentación es un tema que ha suscitado un gran interés en estos últimos años en el ámbito educativo, ya que es una información importante que ayuda al estudiantado a mejorar su trabajo (Brookhart, 2010). Ofrecer una retroalimentación eficaz es una de las tareas más importante en el proceso de aprendizaje y adquisición de una lengua extranjera (LE) o segunda (L2), ya que motiva al estudiantado, lo ayuda y lo guía por medio de acciones necesarias para alcanzar sus objetivos de aprendizaje (Hyland y Hyland, 2006; Mak, 2014; Ramaprasad, 1983; Sadler, 1989).

En el contexto del desarrollo de la competencia de la producción escrita, la retroalimentación es vista como una herramienta esencial y muy útil para ayudar al alumnado a progresar y a mejorarla (Alvira, 2016; Mak, 
2014; Storch y Tapper, 1997). Además, ofrece la posibilidad de tener una atención individualizada de los problemas o de las dificultades que tiene que mejorar y esto no sería posible en las condiciones normales de una clase (Hyland y Hyland, 2001).

En cuanto a las investigaciones sobre la retroalimentación escrita, éstas coinciden sobre la permanencia de la información dada para que pueda ser releída cuantas veces sea necesaria por el estudiantado (Brookhart, 2010; Roberge, 2008; Rodet, 2000). Este tipo de retroalimentación favorece el aprendizaje (Black y Wiliam, 1998; Butler, 1988; Crooks, 1988). Otros estudios concluyen que la retroalimentación permite al estudiantado desarrollar su autonomía, ser más responsables y comprometerse con su aprendizaje, sobre todo cuando se trata de una retroalimentación escrita individualizada (Evans, 2013; Nicol y Macfarlane-Dick, 2006).

Con respeto a la retroalimentación escrita en el aprendizaje de una lengua, por un lado, las investigaciones giran en torno a la importancia de la corrección de errores gramaticales, ya que esto motiva al estudiantado a seguir su aprendizaje, a conocer cuáles son sus errores y a ayudarle a mejorar en la L2 o la LE (Bitchener, Young y Cameron, 2005; Chandler, 2004; Cuxim, 2018; Ferris, 1999; Ferris y Roberts, 2001). Por otro lado, las investigaciones experimentales comparan los efectos de la retroalimentación correctiva directa e indirecta, en las cuales concluyen que la retroalimentación juega un papel esencial en el desarrollo de la producción escrita (Eslami, 2014; Jamalinesari, Rahimi, Gowhary y Azizifar, 2015). Se destacan, además, otras que tratan sobre los tipos de retroalimentación escrita como la de Duijnhouwer, Prins y Stokking (2012) y la de Min (2013), en las cuales identifican que la retroalimentación de tipo constructivo es la más eficaz para el estudiantado, ya que aporta más información para corregir fácilmente sus producciones escritas. También existe una investigación realizada por Sow (2015) con docentes de primaria, en la cual identifica que la de tipo cognitivo fue la más empleada en la producción escrita. Otro grupo importante de investigaciones son las que tratan de las percepciones del profesorado sobre la retroalimentación, como la de García (2013) y Rouleau-Girard (2015). Estas coinciden en que el profesorado dedica más tiempo a la corrección de los trabajos del estudiantado. A pesar de que, en estos últimos años, ha habido numerosas investigaciones sobre la retroalimentación escrita, casi ninguna trata sobre el caso del francés como L2 o LE, la única identificada fue la de Sow (2015) con docentes de primaria. Sin embargo, el autor no indica cuál tipo es más efectivo para el estudiantado ni tampoco investiga sobre la manera en la cual el estudiantado emplea esta retroalimentación.

Por lo tanto, se observa que la retroalimentación es un componente muy importante para el desarrollo de la competencia lingüística de una persona aprendiz de una L2 o LE, ya que, sin ella, es muy difícil que la persona estudiante logre mejorar o aprender de sus errores lingüísticos. Además, sin retroalimentación, se puede correr el riesgo de fosilizarse durante su proceso de aprendizaje, ya que no sabría si lo que está escribiendo está correcto. La retroalimentación es una información que ayuda a prolongar el aprendizaje del estudiantado, a invitarlo a revisar sus producciones, a evitar de cometer los mismos errores en otros trabajos. De esta manera, es esencial seguir investigando sobre esta temática, sobre el uso que el estudiantado da a la retroalimentación y sobre los tipos de retroalimentación que el profesorado ofrece para conocer a profundidad si el tipo de retroalimentación influye en la producción escrita del estudiantado. Por eso, esta investigación busca, por un lado, identificar los tipos de retroalimentación escrita dada por el profesorado y, por otro, analizar la manera en que el estudiantado utilizó la retroalimentación escrita recibida.

En este artículo, se presentan, primeramente, los fundamentos teóricos de la producción escrita en el aprendizaje de una L2 o LE para continuar con una definición del concepto de retroalimentación, así como sus tipos según el modelo de Rodet. Luego, se explica el tipo de investigación, así como los instrumentos y el procedimiento de recolección de datos. Posteriormente, se realiza una descripción y una discusión de los resultados. Finalmente, se presentan las conclusiones y las recomendaciones. 


\section{LA PRODUCCIÓN ESCRITA}

La producción escrita es considerada una competencia lingüística que consiste en formular por escrito un mensaje que se desea comunicar por medio de signos gráficos de una lengua (Cuq y Gruca, 2005; Robert, 2002). Esta competencia lingüística es una actividad difícil y compleja, ya que es la última que adquiere una persona aprendiz de una L2 o de una LE (Rakedson y Baram-Tsabari, 2016). En el ámbito de la enseñanza de una L2 y de una LE, la producción escrita ha ocupado siempre un lugar secundario y tiende a ser desarrollada en los niveles avanzados (Beacco, 2007). En efecto, durante el método de gramáticatraducción, el estudiantado realizaba traducciones de textos como ejercicio de producción escrita (Brown y Abeywickrama, 2010; Lussier y Turner, 1995). Luego, se priorizó la producción oral con el método directo, el método audio-oral, el método estructura-global audiovisual, en los cuales la escritura era solamente un reflejo del lenguaje hablado y desarrollado después de que la persona aprendiz adquiera su competencia oral (Germain, 1993). Finalmente, con la llegada del enfoque comunicativo y de la perspectiva centrada en la acción, se priorizan las cuatro competencias lingüísticas en un contexto auténtico de comunicación (LarsenFreeman y Anderson, 2016). De esta manera, la producción escrita adquiere un papel importante en el aprendizaje de una L2 o una LE y se busca que la persona aprendiz desarrolle esta competencia lingüística, así como el aprendizaje de escritura de diferentes tipos de textos, pasando de lo más simple como el caso de un formulario de inscripción a textos complejos como relatos, cartas postales, argumentaciones, síntesis, disertaciones, entre otros (Chiss, 2012).

En la actualidad, la producción escrita ha tenido un lugar más importante gracias a la comunicación escrita en tiempo real o diferido como el correo, los mensajes de textos, la formación a distancia, etc. (Beacco, 2007). Es una actividad intelectual compleja, ya que implica la movilización de operaciones mentales asociadas al proceso de escritura (la planificación, la escritura del texto, la revisión y la corrección) y a otras operaciones como la organización y la creación de las ideas, la escogencia del léxico, la coherencia y la cohesión del texto, las convenciones lingüísticas, etc. (Culham, 2015; Hayes y Flower, 1980). En el contexto de una L2 y de una LE en los adultos, la competencia lingüística es menos desarrollada que en una L1, ya que existe la presencia de errores, el uso de un léxico más redundante, el uso de una sintaxis más simple y la competencia pragmática es menos desarrollada (Manchón, Roca de Larios y Murphy, 2000).

Manchón (2011) habla de dos enfoques ligados al aprendizaje de la producción escrita: escribir para aprender a escribir (learning to write) y escribir para aprender la lengua (writing to learn). La primera hace referencia sobre el aprendizaje del proceso de escritura, sobre la adquisición de las competencias comunicativas y sobre las convenciones sociales de la escritura. La segunda se refiere a la utilización de la escritura para promover el aprendizaje de una lengua, es decir ayudar al estudiantado a desarrollar las convenciones lingüísticas para la producción escrita. De esta manera, la producción escrita es un medio para aprender una lengua. Por lo tanto, una retroalimentación eficaz es una herramienta importante para ayudar al estudiantado a progresar y a mejorar su producción escrita, tanto a nivel del proceso de escritura como el desarrollo de las convenciones lingüísticas de una lengua como la morfología, la sintaxis, la ortografía, entre otros.

\section{La retroalimentación}

El concepto de retroalimentación proviene de la palabra del inglés feedback. Se comprende como una información ofrecida por una fuente, no necesariamente externa (persona profesora, estudiante, libro, etc.) sobre un aspecto o algunos aspectos de la realización de una persona estudiante y sobre su proceso de aprendizaje, permitiéndole mejorarlo (Bailey y Garner, 2010; Bitchener, 2008; Duijnhouwer et al., 2012; Ferris y Roberts, 2001; Hattie y Timperley, 2007). 
Permite al estudiantado saber lo que se espera de su trabajo, lo que se debe mejorar y lo que está correcta o incorrectamente hecho (Guénette, 2010; Simard, 1999; Valdivia, 2014; Wiggins, 1998). Ayuda a orientar la corrección del trabajo de la persona estudiante (Arancibia, Tapia-Ladino y Correa, 2019; Chandler, 2003; Bernal, 2008; Ferris,1999; Sheen, 2011). Es un diálogo pedagógico entre la persona docente y estudiante (Halté,1984; Miedzo y Chipo, 2019; Morrissette,1999).

Esta información permite al estudiantado prolongar su aprendizaje y consolidar gradualmente las habilidades que se adquirirán progresivamente (Contreras y Zúñiga, 2018; Scallon, 2008). Le permite hacer una revisión de lo que está adquiriendo durante su aprendizaje y ayudarlo en el desarrollo de su proceso de aprendizaje, ya que se le indica lo que ha hecho bien o mal para que pueda hacer ajustes o modificaciones en su trabajo (Bachy y Lebrun, 2009; Hyland y Hyland, 2006; Fithriani, 2019; Lee, 2007; Lee, 2019). Es una fuente de motivación para el estudiantado (Hyland y Hyland, 2006; Chandler, 2004; Ferris, 1999; Kluger y DeNisi, 1996; Voelkel, Varga-Atkins y Mello, 2019; Vollmeyer y Rheinberg, 2015).

Por lo tanto, se concluye que la retroalimentación es una información dada a una persona estudiante por el profesorado o su par, permitiéndole prolongar su aprendizaje, saber lo que ha adquirido, hacer los ajustes o las modificaciones necesarias en sus trabajos, corregirlos, reflexionar su aprendizaje y profundizar sus conocimientos por medio de pistas o indicaciones para lograrlo.

\section{LOS TIPOS DE RETROALIMENTACIÓN ESCRITA}

Existen diferentes tipologías sobre la clasificación de la retroalimentación escrita como la de Arancibia et al. (2019), Brookhart (2010), Halté (1984), Hattie y Timperley (2007) y Leboeuf (1999). Para esta investigación, se utilizará la clasificación de Rodet (2000), ya que es más exhaustiva y clasifica la retroalimentación según cuatro parámetros: (1) los contenidos, (2) la formulación, (3) la intención y (4) los cambios provocados en la persona aprendiz.

De acuerdo con los contenidos, existen cuatro tipos:

a. La retroalimentación de tipo cognitivo. Indica la corrección de los errores, indica lo que hace falta e indica lo que está bien.

b. La retroalimentación de tipo metacognitivo. Realiza preguntas al estudiantado sobre el proceso que ha utilizado y lo lleva a reflexionar sobre los procesos futuros con respecto a la tarea por realizar.

c. La retroalimentación de tipo metodológico. Se refiere a las intervenciones de la persona docente para reforzar la pertinencia de la organización de un procedimiento utilizado, haciendo énfasis en la estructuración de las etapas de realización, así como la utilización de cuadros o de esquemas.

d. La retroalimentación de tipo afectivo. Se refiere a aquellas que ofrecen elementos de motivación o de ánimo al estudiantado.

La retroalimentación puede adoptar tres tipos de formulación:

a. La retroalimentación positiva. Se refiere a aquella que resalta lo que está bien o la que ofrece un mensaje con connotación positiva sin aportar ninguna otra pista.

b. La retroalimentación negativa. Se refiere a aquella que tiene una connotación negativa o peyorativa.

c. La retroalimentación constructiva. Es aquella que invita al estudiantado a profundizar sus conocimientos, invita al diálogo entre la persona docente y estudiante y toma a menudo la forma de una sugerencia.

Con respecto a la intención de la retroalimentación, se distinguen tres tipos:

a. Prescriptiva. Es cuando el profesorado quiere corregir un error, recuerda la regla al estudiantado o aporta una información precisa.

b. Informativa. Es cuando el profesorado transmite un complemento de información para profundizar un tema e indica otras estrategias que podrían haber sido utilizadas con el fin de ayudar a la persona aprendiz. 
c. Sugestiva. Es cuando el profesorado invita al estudiantado a profundizar sus conocimientos por medio del cuestionamiento.

La retroalimentación puede generar tres tipos de cambios en el estudiantado:

a. Una progresión. Es cuando el estudiantado sigue su aprendizaje después de la intervención del profesorado.

b. Una regresión. Es cuando el estudiantado sigue en la incomprensión de su problema.

c. Una neutralidad. Es cuando no se observa ningún tipo de cambio en el estudiantado.

\section{TiPo DE INVESTIGACióN}

Esta investigación se enmarca en el paradigma naturalista de naturaleza cualitativa, partiendo del principio en que, según Fortin (2010), la realidad social es múltiple y se construye a partir de percepciones individuales. Esta investigación es cualitativa de tipo colaborativa, porque busca explorar, comprender y describir un fenómeno según el punto de vista de las personas participantes, según las experiencias vividas y además, existe una implicación de las personas investigadoras con sus participantes y con su medio. La investigación colaborativa tiene como objetivo transformar las maneras de pensar y de hacer de las personas participantes, implicándolas en la investigación y a través de la formación (Boavida y da Ponte, 2011; Bourassa y Boudjaoui, 2012; Desgagné, Bednarz, Couture, Poirier y Lebuis, 2001). Esta situación busca que la persona investigadora y las participantes trabajen estrechamente y en cooperación mutua. Por lo tanto, se define un aspecto que es de interés común entre la persona investigadora y las participantes (Desgagné, 1997; Morrissette, 2013). Para el caso de esta investigación, el aspecto de interés para ambas partes fue el tema de la retroalimentación. Existe una co-construcción de un objeto de conocimiento entre la persona investigadora y las participantes (Desgagné, 1997; Domínguez-Gaona, Crhová y Molina-Landeros, 2015). Para esta coconstrucción, se identificaron las prácticas de retroalimentación que existían de las participantes antes de iniciar la investigación. A partir de esta identificación, se construyeron los diferentes talleres de capacitación para las participantes sobre la temática de la retroalimentación. Después de la implementación de los talleres, hubo un periodo de acompañamiento de una de las personas investigadoras a las participantes durante la retroalimentación de las primeras producciones del estudiantado. Luego, se disminuyó este acompañamiento en los siguientes meses para que las participantes siguieran trabajando de manera más independiente y autónoma, integrando las nuevas prácticas de retroalimentación en sus cursos. La actividad reflexiva de las personas participantes sería el objeto de análisis de la persona investigadora (Durand y Poirier, 2012). Para la actividad reflexiva, hubo varios momentos. Se realizaron reuniones mensuales con las participantes para conocer su percepción sobre las nuevas prácticas de retroalimentación. Por último, hubo una entrevista final a las participantes al término de la investigación. En este artículo, no se presenta la información de la percepción de las prácticas de retroalimentación y de la experiencia vivida por las participantes docentes de esta investigación, sino solamente los tipos de retroalimentación empleados en las producciones escritas.

\section{CONTEXTO Y PARTICIPANTES DE LA INVESTIGACIÓN}

La investigación tuvo lugar en la Escuela de Lenguas Modernas de la Universidad de Costa Rica, específicamente en la sección de francés. Se desarrolló en los cursos de expresión escrita de segundo y tercer año de las carreras de Bachillerato en Francés y Bachillerato en la Enseñanza del Francés.

Con respecto a las personas participantes, fueron dos grupos: tres docentes de los cursos de expresión escrita (dos de segundo año y una de tercer año) y tres grupos de estudiantes. La P1 tiene una maestría en diplomacia y una licenciatura en literatura francesa, tiene más de 40 años y más de diez años de laborar para la carrera. La P2 tiene una maestría en literatura francesa, tiene menos de 40 años y menos de 10 años de laborar 
para la carrera. La P3 tiene una maestría en la enseñanza del francés, tiene más de 50 años y más de 15 años de laborar para la carrera. El grupo de estudiantado lo conforman las personas matriculadas en los cursos de estas tres docentes. En la tabla 1 se describen las características del estudiantado.

TABLA 1

Las características del estudiantado de los cursos de expresión escrita de la Escuela de Lenguas Modernas

\begin{tabular}{|c|c|c|c|}
\hline Caracteristicas & $\begin{array}{l}\text { Segundo } \\
\text { año Grupo } 1 \mathrm{~N} \text { : } \\
17\end{array}$ & $\begin{array}{l}\text { Segundo } \\
\text { año Grupo } 2 \mathrm{~N} \text { : } \\
18\end{array}$ & $\begin{array}{l}\text { Tercer } \\
\text { año Grupo } 3 \mathrm{~N} \text { : } \\
13\end{array}$ \\
\hline Género & $\begin{array}{l}12 \text { mujeres } 5 \\
\text { hombres }\end{array}$ & $\begin{array}{l}15 \text { mujeres } 3 \\
\text { hombres }\end{array}$ & $\begin{array}{l}10 \text { mujeres } 3 \\
\text { hombres }\end{array}$ \\
\hline Edad & $\begin{array}{l}10 \text { de } 18 \text { a } 21 \\
\text { años } 7 \text { de } 22 \\
\text { años o más }\end{array}$ & $\begin{array}{l}8 \text { de } 18 \text { a } 21 \\
\text { años } 10 \text { de } 22 \\
\text { años } 0 \text { más }\end{array}$ & $\begin{array}{l}9 \text { de } 18 \text { a } 21 \\
\text { años } 4 \text { de } 22 \\
\text { años o más }\end{array}$ \\
\hline $\begin{array}{l}\text { Aprendizaje del } \\
\text { francés }\end{array}$ & $\begin{array}{l}10 \text { en la } \\
\text { universidad } 1 \\
\text { en la escuela } 6 \\
\text { en el colegio }\end{array}$ & $\begin{array}{l}9 \text { en la } \\
\text { universidad } 3 \\
\text { en la escuela } 6 \\
\text { en el colegio }\end{array}$ & $\begin{array}{l}11 \text { en la } \\
\text { universidad } 1 \\
\text { en el colegio } 1 \\
\text { en Francia }\end{array}$ \\
\hline
\end{tabular}

Fuente: Elaboración propia, 2020.

\section{LAS TÉCNICAS Y LOS INSTRUMENTOS DE RECOLECCIÓN DE DATOS}

Las preguntas de la guía de entrevista para el grupo de discusión fueron validadas por seis personas expertas de la Universidad de Montreal y dos personas expertas de la Universidad de Costa Rica. También, para validar las preguntas a las personas participantes, se realizó una entrevista a dos personas estudiantes no participantes en la investigación con el fin de ver si las guías de entrevista permitían recoger los datos necesarios para la investigación. Todos los grupos de discusión fueron realizados por una persona del equipo investigador quien no conocía a las personas estudiantes ni había sido docente en los cursos.

Los grupos de discusión se realizaron en dos etapas diferentes con el estudiantado de cada grupo. El grupo 1 estuvo integrado por la población estudiantil de la P1, el grupo 2 por la de la P2 y el grupo 3 por la de la P3. El primer momento de grupo de discusión con cada grupo de estudiantes se realizó en el mes de julio de 2016. El segundo momento fue en el mes de diciembre de 2016. Las transcripciones de las entrevistas y de los grupos de discusión fueron realizadas a lo largo del segundo semestre de 2016 y del primer semestre de 2017.

En el mes de julio de 2016, se desarrollaron dos talleres sobre la retroalimentación para el personal docente a partir de las prácticas identificadas en los grupos de discusión y se utilizó la tipología de Rodet (2000). Una persona del equipo investigador impartió los dos talleres.

\section{Las producciones escritas revisadas por las tres docentes}

Se fotocopiaron los trabajos corregidos por las tres docentes. En el caso de la P1, hubo seis producciones escritas a lo largo del semestre y se recolectaron 89 trabajos producidos por el estudiantado. En el caso de la P2, hubo ocho producciones escritas a lo largo del semestre y se recolectaron 137 trabajos. Por último, para el caso de la P3, el estudiantado escribió nueve producciones escritas y se recogieron en total 107 trabajos. Para el caso de la P1, se identificaron 100 retroalimentaciones escritas. La P2 escribió 320 retroalimentaciones escritas y la P3 escribió 263. 


\section{El tratamiento y análisis de los datos}

El análisis de datos obtenidos en los grupos de discusión fue realizado por el equipo investigador y se usó el programa de $Q D A$ Miner para analizar el contenido según los códigos temáticos preestablecidos. Además, hubo un contracodaje; esta técnica consiste en la confrontación de los códigos con varias personas codificadoras (Van der Maren, 1996). Esto ayudó a garantizar la fiabilidad del análisis de los datos.

Una especialista de la Universidad de Montreal y otra de la Universidad de Costa Rica realizaron el contracodaje de los grupos de discusión. Esto se realizó durante los meses de abril y mayo de 2017. La concordancia entre la codificación del equipo investigador y de las especialistas fue de un 98,12\% para los grupos de discusión.

En relación con la codificación de la retroalimentación escrita identificada, se realizó también un análisis de contenido y se utilizó la tipología de Rodet (2000). Para esta tarea, una de las personas investigadoras realizó una primera codificación en un documento Word y quince días después hizo una segunda codificación con el programa QDA Miner. La codificación fue realizada en el mes de junio de 2017.

Se realizó además un contracodaje para la retroalimentación escrita. Esto fue realizado por una especialista en la Universidad de Montreal. Se obtuvo un resultado de $84,91 \%$ de concordancia.

\section{LIMITACIONES DE LA INVESTIGACIÓN}

Por ser una investigación de tipo cualitativo colaborativo, no se puede hacer una generalización de los resultados por el número limitado de participantes, sobre todo el caso de las docentes. Otra limitación está relacionada con la cantidad de producciones escritas en cada grupo. En un principio, se había pensado que, en segundo año, cada estudiante realizara ocho producciones escritas, pero no se pudo concretar, ya que en el grupo de la P1 solamente se logró que cada estudiante escribiera seis.

Por último, la otra dificultad que hubo fue que todo el estudiantado cumpliera con la cantidad de producciones escritas establecidas en cada grupo. Sin embargo, esto tampoco se logró, ya que hubo estudiantes que no entregaron algunas de las producciones escritas. Por lo tanto, no fue posible observar si hubo un progreso, un retroceso o una neutralidad en cuanto al desarrollo de la competencia de la producción escrita al final del semestre.

Como síntesis de esta sección, es importante mencionar que este estudio tiene la característica de una investigación cualitativa de tipo colaborativa, en la cual las personas investigadoras trabajan en conjunto con las participantes a partir de un tema de interés de ambas partes. Se utilizaron como principal técnica de recolección de datos la entrevista en los grupos de discusión y el análisis de las producciones escritas del estudiantado. A pesar de las limitaciones encontradas, se obtuvo suficiente información sobre la práctica de retroalimentación de las participantes y sobre la percepción del estudiantado acerca de esta, que serán descritas y discutidas en la siguiente sección.

\section{ANÁLISIS Y DISCUSIÓN DE LOS RESULTADOS}

En esta sección se expone, primeramente, la tipología de la retroalimentación ofrecida por el cuerpo docente al estudiantado. Luego, se presentan las percepciones del estudiantado hacia las retroalimentaciones recibidas.

\section{Los tipos de retroalimentación escrita de las participantes}

El primer objetivo de esta investigación busca identificar los tipos de retroalimentación escrita ofrecida por el cuerpo docente a sus estudiantes. Rodet (2000) propone un modelo de tres parámetros para categorizar 
la retroalimentación escrita, a saber, los contenidos (cognitivo, metacognitivo, metodológico y afectivo), la formulación (positiva, negativa y constructiva) y la intención (prescriptiva, informativa y sugestiva). La P1 formuló en total 100 retroalimentaciones escritas. Según el contenido, el más utilizado fue el de tipo cognitivo ( $\mathrm{N}=77$, equivalente a un $77 \%)$, por ejemplo: debe corregir el registro de la lengua. Hubo 18 casos de tipo metodológico (18\%), por ejemplo: falta una parte de la transición para el siguiente párrafo. Las de tipo metacognitivo $(\mathrm{N}=3$, equivalente a un $3 \%)$ y de tipo afectivo $(\mathrm{N}=2$, equivalente a un $2 \%)$ fueron las menos empleadas, por ejemplo: ¿Cuál tipo de música se refiere y en dónde está esta idea? y Buen trabajo! En cuanto a la formulación, la P1 utilizó a menudo la de tipo positivo ( $\mathrm{N}=55$, equivalente a un 55\%), por ejemplo: ¿En dónde está el anuncio del plan? Hubo 36 casos (36\%) de tipo constructivo, por ejemplo: Atención usted tiene buenas ideas, pero tenga cuidado para evitar abordar otro tema. La de tipo negativo fue la menos utilizada con 9 casos (9\%), por ejemplo: ¡Fuera de tema! Con respecto a la intención, la de tipo informativo fue la más usada ( $\mathrm{N}=68$, equivalente a un 68\%), por ejemplo: ¡Ponga atención a los determinantes! Hubo también 19 casos (19\%) de tipo prescriptivo, por ejemplo: Retrabaje esta idea, la idea tiene que estar relacionada con el tema desarrollado. Finalmente, hubo 13 casos (13\%) de tipo sugestivo, por ejemplo: ¿La palabra empresa es de género masculino o femenino en francés, podría utilizar un diccionario para verificar el género?

El resultado obtenido en la retroalimentación escrita ofrecida por la P1 hace pensar que las correcciones de los errores del estudiantado tienen un papel importante en las prácticas de esta participante. Por eso, existe una gran mayoría de tipo cognitivo y casi ninguno de tipo metacognitivo, que tiene como objetivo que el estudiantado reflexione sobre sus errores y sobre su aprendizaje. Esto refleja que la P1 prioriza sobre todo la producción escrita como una herramienta para que el estudiantado aprenda la lengua. Si se observa el tipo de formulación, existe una prioridad sobre lo positivo. La retroalimentación de tipo positivo sirve sobre todo para mantener la motivación del estudiantado, sin aportarle una información precisa sobre lo que debe trabajar o corregir. Por último, la P1 prefiere en cuanto al contenido la de tipo informativo. Este tipo de retroalimentación ayuda al estudiantado a conocer lo que tiene que poner atención en sus próximos trabajos. Esto es de gran utilidad, ya que le ayuda a enfocarse en lo que se debe mejorar la próxima vez.

En el caso de la P2, se identificaron 320 retroalimentaciones escritas en las producciones escritas del estudiantado. En cuanto al contenido, formuló frecuentemente la de tipo cognitivo $(\mathrm{N}=184$, equivalente a un 57,5\%), por ejemplo: ponga atención en las faltas de ortografía. Seguidamente, hubo 79 casos (24,7\%) de tipo metodológico, por ejemplo: haga un plan de la redacción antes de empezar a escribir. Hubo también 43 casos $(13,4 \%)$ de tipo afectivo, por ejemplo: ¡fácil de leer, ideas claras, muy bien! Por último, la de tipo metacognitivo fue la menos utilizada $(\mathrm{N}=14$, equivalente a un 4,4\%), por ejemplo: ¿Cómo hizo para hacer las transiciones entre una idea a otra? Con respecto a la formulación, la de tipo constructivo fue la más usada ( $\mathrm{N}=152$, equivalente a un $47,5 \%)$, por ejemplo: podría usar sinónimos y pronombres para evitar la repetición. La de tipo negativo fue identificada en 89 casos $(27,8 \%)$, por ejemplo: el párrafo no corresponde a la pregunta planteada. La de tipo positivo fue encontrado en 79 casos $(24,7 \%)$, por ejemplo: es un párrafo bien estructurado y organizado, las ideas siguen un orden lógico. Con relación a la intención, la de tipo informativo fue la más utilizada $(\mathrm{N}=237$, equivalente a un $74,1 \%)$, por ejemplo: las ideas tienen una estructura del español. La de tipo prescriptivo fue encontrada en 46 casos (14,4\%), por ejemplo: en la oración francesa, el verbo debe estar al lado del sujeto, reorganice los elementos de su oración. Por último, la de tipo sugestivo fue identificada en 37 casos (11,6\%), por ejemplo: ¿Podría usar oraciones simples con una construcción concisa?

$\mathrm{Al}$ observar las características de la retroalimentación ofrecida por la P2, es importante señalar también que el tipo cognitivo es el más empleado. Esto refleja de nuevo el interés de la P2 por corregir los errores de sus estudiantes y toma la producción escrita como un medio para aprender la lengua. Se destaca también la de tipo constructivo por su formulación. Esta situación es muy interesante, ya que las investigaciones muestran que la retroalimentación de tipo constructivo ayuda mejor al estudiantado a corregir sus errores y aporta una información más pertinente que indicar solamente si el trabajo está bien o mal. La retroalimentación constructiva es muy útil, porque guía mejor en la corrección de la persona estudiante y aporta una 
información más clara sobre lo que tiene que mejorar o corregir. Además, este tipo de retroalimentación aporta una información precisa sobre lo que tiene que hacer para sus próximos trabajos. Por lo tanto, se podría decir que la P2 busca guiar mejor a su estudiantado durante sus trabajos. Es por eso que, en su caso, hubo mucha más retroalimentación que la P1. En cuanto al contenido, estas coinciden con las de la P1, ya que la mayoría son de tipo informativo.

En cuanto a la P3, se identificaron 263 casos. Según el contenido, hubo 145 casos (55,1\%) de tipo cognitivo, por ejemplo: ponga atención en las concordancias entre el sustantivo y el adjetivo. Seguidamente, hubo 85 casos $(32,3 \%)$ de tipo metodológico, por ejemplo: sería necesario dividir el texto en dos párrafos. Hubo 21 casos (8\%) de tipo afectivo, por ejemplo: muy buena organización del texto. Hubo 12 casos (4,6\%) de tipo metacognitivo, por ejemplo: ¿Cuáles son las ideas claves para hacer el informe? Con respecto a la formulación, la de tipo constructivo fue la más empleada (179 casos, equivalente a un 68,4\%), por ejemplo: ¿En la lengua escrita, se puede eliminar el adverbio de negación? La de tipo positivo fue identificada en 45 casos (16,7\%), por ejemplo: esta idea está ligada al tercer párrafo. La de tipo negativo fue encontrada en 39 casos (14,8\%), por ejemplo: no se puede dar su opinión. Por último, en relación con la intención, la de tipo informativo fue la más utilizada ( $\mathrm{N}=124$, equivalente a un $47,1 \%)$, por ejemplo: esta oración fue tomada del texto. La de tipo prescriptivo fue encontrada en 110 casos (41,8\%), por ejemplo: cuando comienza un párrafo, es necesario dejar un espacio. La menos utilizada fue la de tipo sugestivo $(\mathrm{N}=29$, equivalente a un $11 \%)$, por ejemplo: ¿Utilizó un diccionario de sinónimos para evitar la repetición?

Al observar la retroalimentación dada por la P3, existe también una preferencia por la de tipo cognitivo, priorizando de nuevo la importancia de la corrección de errores y utilizando la producción escrita como un medio para aprender la lengua. Sin embargo, existe una tendencia también a utilizar la de tipo metodológico. Esto es importante de señalar, ya que en su caso también se usa la producción escrita como un medio para aprender el proceso de escritura a diferencia de las otras dos participantes, en donde no era muy frecuente el uso de la retroalimentación de tipo metodológico. Esta participante, al igual que la P2, tiene una preferencia por la de tipo constructivo. Esto ayuda mejor a la persona aprendiz a corregir y a mejorar su producción escrita, ya que aclara mejor sobre lo que tiene que trabajar de nuevo. Además, la cantidad de retroalimentación ofrecida por esta participante es muy parecida a la P2. Por lo tanto, se podría decir que la P3 también buscar guiar de la mejor manera a sus estudiantes durante la producción escrita. De nuevo, la de tipo informativo es la más empleada, coincidiendo con las otras dos participantes.

En esta investigación se encontró que las tres docentes formularon sobre todo la retroalimentación escrita de tipo cognitivo en cuanto a contenido, de tipo constructivo para la P2 y la P3, y de tipo positivo para la P1 en cuanto a la formulación, y de tipo informativo en cuanto a la intención. Esta situación coincide con los resultados obtenidos en la investigación cualitativa de Sow (2015) con docentes de primaria, utilizando la misma tipología para clasificar la retroalimentación escrita en las producciones escritas, ya que, en su estudio, el investigador encontró que sus participantes tienden a formular una retroalimentación de tipo cognitivo, en la cual se señala el error a su estudiantado y se le indica lo que se debe corregir y así asegurar la comprensión de sus estudiantes. Identificó también que la formulación más utilizada fue la de tipo positivo. Sin embargo, este autor no realizó la clasificación según la intención de la retroalimentación escrita.

Otras investigaciones colaborativas utilizando esta misma tipología fueron la de Lapointe (2011) en el campo de las matemáticas y la de Durand y Poirier (2012) en el campo de la lectura con docentes de primaria. Ambas investigaciones identificaron que la de tipo metodológico fue la más utilizada. Esta situación contradice a los resultados obtenidos en esta investigación. Esto se podría explicar por el hecho de que, en estas disciplinas, el interés de las investigadoras se centra sobre todo en el procedimiento de la resolución de problemas en matemáticas y en las estrategias aplicadas a la lectura. En cambio, en una disciplina como el aprendizaje de una lengua, la de tipo cognitivo fue la más utilizada, ya que el interés se centra sobre todo en la corrección de los errores lingüísticos para evitar que las personas aprendices repitan sus errores en nuevos trabajos. Sin embargo, también se encontraron retroalimentaciones de tipo metodológico en esta 
investigación, sobre todo las que implicaban el uso de un plan de redacción para estructurar las ideas y los ejemplos asociados a ellas. Por lo tanto, es importante mencionar que la retroalimentación ofrecida por las participantes coincide sobre todo con el enfoque de escribir para aprender la lengua, ya que existe un interés de señalar y de corregir los errores lingüísticos. Además, según el tipo de retroalimentación dada, se podría pensar que dos de las participantes buscan guiar de manera más clara al estudiantado para que estos eviten repetir los mismos problemas en sus producciones.

Al comparar la retroalimentación escrita combinando los tres parámetros de las tres participantes, se destaca que las tres usan la de tipo cognitivo constructivo informativo. Esto se podría explicar, ya que existe una preferencia por corregir los errores al estudiantado, aportándole la información correcta con el fin de que no cometa el mismo tipo de error la próxima vez. También esta situación estaría ligada al enfoque pedagógico más tradicional de la enseñanza de una L2 o una LE, en el cual la persona docente es el centro de todo el proceso de aprendizaje y enseñanza de la L2 o LE. Por lo tanto, tiene que aportar la respuesta a los errores del estudiantado. Es por eso que en las tres participantes se identificó muy poca retroalimentación de tipo metacognitivo, la cual busca que la persona aprendiz reflexione sobre sus propios procesos de aprendizaje y tenga una mayor autonomía.

Si se observan las particularidades de cada participante, la P1 tiene también una preferencia por las de tipo cognitivo positivo informativo y prescriptivo, la P2 prefiere también las de tipo afectivo positivo informativo y cognitivo negativo informativo, y la P3 usa también la de tipo metodológico constructivo prescriptivo y cognitivo constructivo prescriptivo. Para el caso de la P3, esto haría suponer que el tipo de producción escrita realizada por el estudiantado tuvo una influencia en la retroalimentación dada, ya que el estudiantado del tercer año escribe sobre todo producciones que requieren respetar algunas reglas de organización del texto. Esto podría haber influido en la retroalimentación dada por la P3. En el caso de la P1 y de la P2, ambas participantes impartían el mismo curso y trabajaban la misma técnica de producción escrita. Es difícil de explicar la poca coincidencia en cuanto al tipo de retroalimentación dada. Se podría pensar que las características individuales como la experiencia en la enseñanza, la formación académica o las creencias que ambas tenían influenciaron en los tipos de retroalimentación.

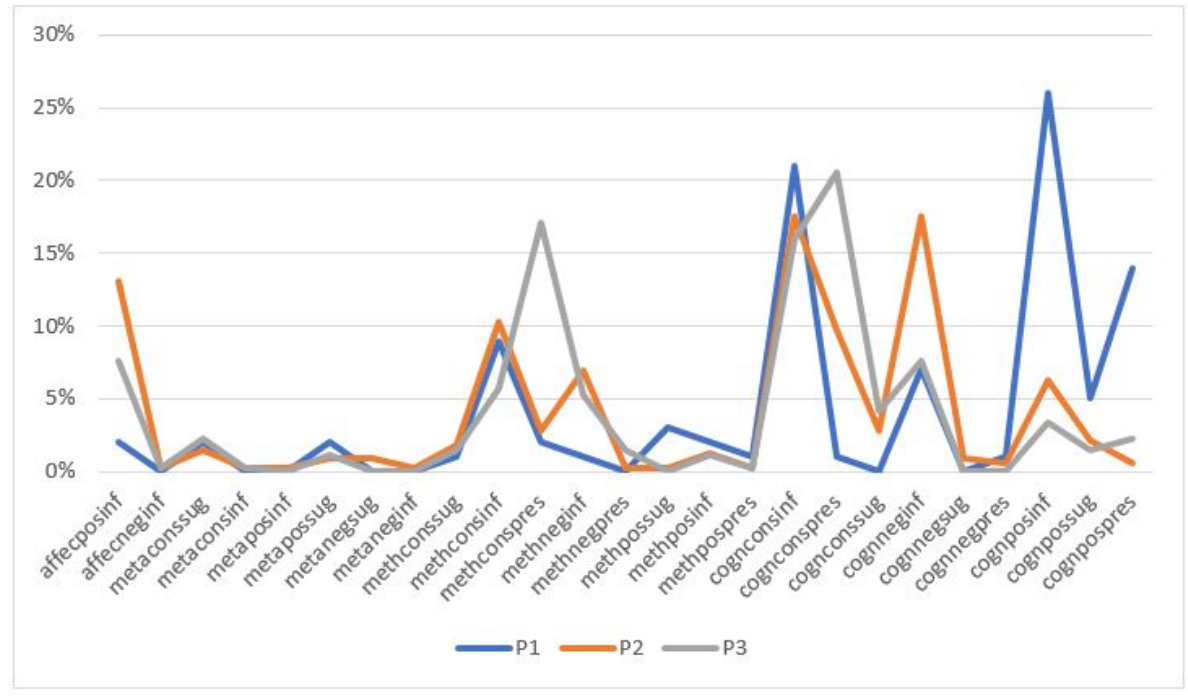

FIGURA 1.

Frecuencia de la retroalimentación escrita según los tres parámetros combinados de las tres participantes Fuente: Elaboración propia, 2020.

Como síntesis de esta sección, se identificaron en total 683 (Figura 1) retroalimentaciones escritas en las producciones escritas corregidas por las tres docentes. Todos los tipos de retroalimentación fueron identificados en las prácticas de las tres docentes. Las de contenido de tipo cognitivo fueron las que más se 
utilizaron ( $\mathrm{N}=406$, equivalente a un $59,4 \%)$, seguidas de las de tipo metodológico $(\mathrm{N}=182$, equivalente a un 26,6\%). Las menos utilizadas fueron las de tipo afectivo ( $\mathrm{N}=66$, equivalente a un 9,7\%) y las de tipo metacognitivo $(\mathrm{N}=29$, equivalente a un 4,2\%). En cuanto a la formulación, los tres tipos estaban presentes en las prácticas de las tres docentes, de las cuales la de tipo constructivo fue la más empleada $(\mathrm{N}=367$, equivalente a un $53,7 \%)$, seguida de la de tipo positivo $(\mathrm{N}=179$, equivalente a un $26,3 \%)$ y, por último, la de tipo negativo $(\mathrm{N}=137$, equivalente a un $20 \%)$. Por último, según la intención de la retroalimentación, los tres tipos estaban presentes en las prácticas de las docentes. La de tipo informativo fue la más empleada $(\mathrm{N}=433$, equivalente a un $63,3 \%)$, seguida de la de tipo prescriptivo ( $N=170$, equivalente a un $24,9 \%)$ y, finalmente, la de tipo sugestivo $(\mathrm{N}=80$, equivalente a un $12 \%)$. Por último, se observa también que la retroalimentación ofrecida por la P2 y la P3 es mucho mayor que la P1. Esta situación muestra que existe una mayor preocupación por parte de la P2 y de la P3 para guiar a su estudiantado durante sus producciones escritas.

\section{El uso de la retroalimentación recibida por el estudiantado}

El segundo objetivo de la investigación es analizar la manera en que el estudiantado utilizó la retroalimentación escrita recibida. Es necesario recordar que la retroalimentación es una información ofrecida por una persona docente, en este caso, al trabajo de una persona estudiante con el fin de ayudarla a corregir o revisar su producción escrita (Arancibia et al., 2019; Brookhart, 2010; Roberge, 2008).

El estudiantado utilizó de diferentes maneras las retroalimentaciones escritas dadas por su profesora. Primeramente, indicó que fue una herramienta útil para hacer la corrección de los errores lingüísticos y comunicacionales. Efectivamente, para la persona estudiante GP2-E4, esto le ayudó a corregir los errores cometidos y tratar de no cometerlos de nuevo (GP2-E4, comunicación personal, 12 de diciembre de 2016). También sirve para corregir errores de sintaxis como lo indicó la GP1-E1: primero, vi las anotaciones de la profesora para corregir mis errores de sintaxis por ejemplo (GP1-E1, comunicación personal, 14 de diciembre de 2016).

Para otras personas estudiantes, la retroalimentación es una ayuda para realizar una revisión de su trabajo como lo ilustra la GP1-E6: la retroalimentación me ayudaba a revisar mi trabajo, por ejemplo, el vocabulario (GP1-E6, comunicación personal, 14 de diciembre de 2016).

Otras personas indicaron que la retroalimentación les ayudó a incrementar la reflexión en la lengua, por ejemplo: Traté de seguirlas, ver que me proponía, las soluciones que ella me daba, porque a veces yo ponía una frase y ponía intente mejor... o tiene más sentido... o suena más elegante... o falta una palabra o un conector que le dé sentido (GP2-E1, comunicación personal, 12 de diciembre de 2016) y Para corregir los errores, por ejemplo, las ideas, la profe pone esto le falta tal cosa. Entonces me ayuda a corregir esa idea. Por ejemplo, el vocabulario, ella pone changer de verbe, entonces uno busca sinónimos o busca en el diccionario el significado bien de la palabra (GP2-E7, comunicación personal, 12 de diciembre de 2016).

El estudiantado las utilizó para evitar repetir los mismos errores en los próximos trabajos, por ejemplo: poner atención a los errores al momento de hacer los próximos trabajos (GP3-E2, comunicación personal, 15 de diciembre de 2016) o para no repetir el mismo error en la tarea siguiente (GP1-E6, comunicación personal, 14 de diciembre de 2016).

Es importante mencionar también que la retroalimentación es una fuente de motivación para el estudiantado como se ilustra en los verbatims siguientes: motivación para ver en qué me equivoqué y cuando uno lo sabia, si podia corregirlo (GP1-E1, comunicación personal, 14 de diciembre de 2016) y vi que me puso que tenia un buen inicio, eso fue algo que me motivó porque me decía que voy por buen camino (GP1-E5, comunicación personal, 14 de diciembre de 2016). Además, esto le ayudaba a seguir aprendiendo como lo muestra el comentario siguiente: de manera positiva, porque me decia que habia ideas que estaban bien, pero que debia trabajar en la manera de conectarlas (GP1-E3, comunicación personal, 14 de diciembre de 2016). 
La retroalimentación es también una información que guía al estudiantado a corregir sus errores, a progresar y a mejorar como se muestra en los ejemplos siguientes: nos ayudaba a guiarnos durante la redacción, ya que se sabía lo que uno tenía que poner en las oraciones y cuáles palabras se debían poner o no (GP1-E3, comunicación personal, 14 de diciembre de 2016), para mantener el nivel (GP1-E1, comunicación personal, 14 de diciembre de 2016) o para aumentar el nivel de los trabajos (GP1-E6, comunicación personal, 14 de diciembre de 2016).

Efectivamente, el estudiantado señaló que la retroalimentación recibida cumplía con una función redaccional, ya que le permitía observar los errores cometidos y corregirlos. Esto coincide con los resultados obtenidos en las investigaciones de Guénette (2010) y Simard (1999). La retroalimentación escrita es una información utilizada para orientar la corrección de la persona aprendiz (Chandler, 2003; Bernal, 2008; Ferris, 1999; Lee, 2019; Sheen, 2011). Por lo tanto, es importante que la producción escrita tenga una retroalimentación por parte del profesorado, sino no sería útil para el estudiantado, ya que no sabría cómo realizar la corrección ni sabría lo que tendría que mejorar. Es por eso que las investigaciones han señalado que la retroalimentación escrita permitía al estudiantado mejorar sus trabajos (Bailey y Garner, 2010; Bitchener, 2008; Duijnhouver et al., 2012; Ferris y Roberts, 2001; Fithriani, 2019; Hattie y Timperley, 2007; Nicol y Macfarlane-Dick, 2006), como se pudo comprobar también en esta investigación, en la cual el estudiantado afirmó que le permitía realizar una revisión de sus trabajos y mejorarlos según las indicaciones dadas por el profesorado.

Otro elemento mencionado por las investigaciones es el hecho de que la retroalimentación tiene un rol afectivo en el aprendizaje, ya que motiva al estudiantado en ese proceso (Hyland y Hyland, 2006; Chandler, 2004; Ferris, 1999; Voelkel et al., 2019; Vollmeyer y Rheinberg, 2015). Esto también fue identificado en esta investigación, puesto que el estudiantado apreció la manera en la cual el profesorado aportó las informaciones necesarias para trabajar sus producciones escritas.

Por último, la retroalimentación es un recurso para mantener un diálogo pedagógico entre el profesorado y el estudiantado (Halté, 1984; Miedzo y Chipo; 2019; Morrissette, 1999). Esta situación también fue encontrada en esta investigación, puesto que el estudiantado utilizó esta información para evitar cometer los mismos errores en sus trabajos y le permitía saber si estaba bien lo que estaba realizando.

Como síntesis, se puede afirmar que la retroalimentación es una fuente importante de aprendizaje para el estudiantado, ya que le ayuda a identificar lo que tiene que corregir y mejorar. Además, es un recurso que motiva al estudiantado en su aprendizaje. Ahora bien, si no hubiera ninguna retroalimentación en el trabajo del estudiantado, es muy probable que éste no pueda saber lo que tendría que trabajar en sus producciones escritas y esto podría causar una fosilización en su aprendizaje.

\section{Conclusiones}

En esta investigación, se trataron los siguientes objetivos: identificar los tipos de retroalimentación escrita ofrecida por el profesorado al estudiantado y analizar de qué manera el estudiantado empleó esta retroalimentación escrita en sus producciones escritas.

Por un lado, según el modelo de Rodet (2000), se encontró que las tres personas docentes emplearon sobre todo una retroalimentación de tipo cognitivo en relación con el contenido; de tipo informativo con respecto a la intención; de tipo constructivo para la P2 y P3, y de tipo positivo para el caso de la P1 en cuanto a la formulación. Los tipos metacognitivos, negativos y sugestivos eran los menos empleados por la población docente. Por otro lado, se identificó que la retroalimentación cumplía con varias funciones, por ejemplo: corrección de los errores, fuente de motivación, mejora de las producciones escritas, diálogo pedagógico entre el estudiantado y el profesorado, entre otras.

La retroalimentación no es solamente el ofrecer una nota a un trabajo, como se ha hecho tradicionalmente, o decir solamente que el trabajo está bien o está mal, sino, la retroalimentación debe ser una información útil 
para el estudiantado, en la cual se le indica lo que está haciendo bien y lo que tiene que mejorar, aportando las precisiones y las aclaraciones necesarias para que sea en definitiva una retroalimentación eficaz. Es importante que las personas docentes de una L2 o de una LE cambien sus prácticas de retroalimentación y las integren en sus prácticas pedagógicas, ya que esta información es un recurso muy importante para el aprendizaje del estudiantado, sobre todo para el caso del desarrollo de la competencia de la producción escrita en una lengua. Además, esta información guía al estudiantado en su proceso de aprendizaje y le ayuda a conocer lo que está realizando bien y lo que debe corregir. Cumple también con una función redaccional, ya que indica al estudiantado sus errores lingüísticos y comunicativos con el fin de que evite cometer los mismos problemas en los siguientes trabajos. De esta manera, la retroalimentación juega un papel esencial en el proceso de aprendizaje y de adquisición de una lengua. En el caso de la producción escrita, cumple también el papel de los dos enfoques de escritura, es decir, la retroalimentación ayuda a la persona aprendiz a aprender una lengua y a aprender el proceso de escritura.

Por lo tanto, las recomendaciones finales de esta investigación se enfocarían a la integración de las prácticas de retroalimentación en clase, considerando la retroalimentación como una información que prolonga el proceso de aprendizaje del estudiantado y le aporte las herramientas necesarias para mejorar su producción escrita, por ejemplo, indicándole lo que ha realizado bien y cómo podría mejorar sus errores o su redacción. Además, es importante que esta retroalimentación no sea solamente una información que se resuma en una nota o en un solo mensaje positivo, sino que sea un comentario constructivo, describiendo cómo podría corregir sus errores o qué tendría que hacer para mejorar o cómo podría hacer para corregirse y que lo haga reflexionar sobre su propio proceso de escritura y de sus errores. De esta manera, se podría ayudar al estudiantado a desarrollar su autonomía y su responsabilidad y a implicarlo en su propio proceso de aprendizaje. Además, es importante que la retroalimentación sea inmediata para que pueda ser eficaz, ya que, si la persona docente toma mucho tiempo en corregir el trabajo de su estudiante, este último puede olvidarse de lo que escribió o del proceso utilizado para realizar su trabajo. Por lo tanto, si la retroalimentación es inmediata, esta se convierte en un aliado importante para el proceso de aprendizaje del estudiantado. La retroalimentación es una herramienta muy importante y podría contribuir mucho en el proceso de aprendizaje del estudiantado cuando le transmite una información clara y precisa.

Por último, cabe mencionar que sería interesante en futuras investigaciones conocer cuál de los tipos de retroalimentación es más efectivo en el aprendizaje de una lengua, ya que en esta investigación sólo se trató de identificarlos, pero no se investigó si algunos de los tipos tienen un mayor impacto en el proceso de aprendizaje del estudiantado. Además, sería interesante investigar el impacto de la retroalimentación a través de un estudio longitudinal en el aprendizaje del estudiantado, ya que esta investigación se trató solamente de seguir a un grupo de estudiantes durante un semestre. Por lo tanto, no se podría medir el impacto de la retroalimentación a largo plazo.

\section{REFERENCIAS}

Alvira, R. (2016). The impact of oral and written feedback on EFL writers with the use of screencasts. Profile, 18, 79-92. doi: https://doi.org/10.15446/profile.v18n2.53397

Arancibia, B.; Tapia-Ladino, M. y Correa, R. (2019). La retroalimentación durante el proceso de escritura de la tesis en carreras de pedagogía: descripción de los comentarios escritos de los profesores guías. Revista Signos, 52, 243-264. doi: https://doi.org/10.4067/S0718-09342019000200242

Bachy, S. y Lebrun, M. (2009). Catégorisation de techniques de rétroaction pour l'enseignement universitaire. Mesure et évaluation en éducation, 32, 29-47. doi: https://doi.org/10.7202/1024953ar

Bailey, R. y Garner, M. (2010). Is the feedback in higher education assessment worth the paper it is written on? Teachers' reflections on their practices. Teaching in Higher Education, 15, 187-198. doi: https://doi.org/10.10 $80 / 13562511003620019$ 
Beacco, J.-C. (2007). L'approche par compétences dans l'enseignement des langues. Paris : Didier.

Bernal, A.M. (2008). Descripción de la retroalimentación escrita que suministran los profesores del área de francés en la habilidad de escritura a los estudiantes de la licenciatura en lenguas modernas de la Pontificia Universidad Javeriana. (Tesis de Maestría). Pontificia Universidad Javeriana, Colombia.

Bitchener, J. (2008). Evidence in support written corrective feedback. Journal of Second Language Writing, 17, 102118. doi: https://doi.org/10.1016/j.jslw.2007.11.004

Bitchener, J., Young, S. y Cameron, D. (2005). The effect of different types of corrective feedback on ESL student writing. Journal of Second Language Writing, 14(3), 191-205. doi: https://doi.org/10.1016/j.jslw.2005.08.001

Black, P. y Wiliam, D. (1998). Assessment and classroom learning. Assessment in Education: Principles policy and practice, 5, 7-73. doi: http://dx.doi.org/10.1080/0969595980050102

Boavida, A.M. y da Ponte, J.P. (2011). Investigación colaborativa: potencialidades y problemas. Revista Educación y Pedagogía, 23(59), 125 -135. Recuperado de https://www.academia.edu/20946690/Investigaci\%C3\%B3n_co laborativa_potencialidades_y_problemas

Bourassa, B. y Boudjaoui, M. (2012). Des recherches collaboratives en sciences humaines et sociales (SHS) : enjeux, modalités et limites. Québec : Presses de l'Université Laval.

Brookhart, S.M. (2010). La retroaction efficace: des stratégies pour soutenir les élèves dans leur apprentissage. Montréal : Chenelière éducation.

Brown, H. y Abeywickrama, P. (2010). Language Assessment: Principles and Classroom Practices. New York: Pearson Longman.

Butler, R. (1988). Enhancing and undermining intrinsic motivation: the effects of task-involving and ego-involving evaluation on interest and performance. British Journal of Educational Psychology, 58, 1-14. doi: https://doi.or g/10.1111/j.2044-8279.1988.tb00874.x

Chandler, J. (2003). The efficacy of various kind of error feedback for improvement in the accuracy and fluency of L2 student writing. Journal of Second Language Writing, 12, 267-296. doi: https://doi.org/10.1016/S1060-37 43(03)00038-9

Chandler, J. (2004). Dialogue: a response to Truscott. Journal of second language writing, 13, 345-348. doi: https:// doi.org/10.1016/j.jslw.2004.05.001

Chiss, J.-L. (2012). L'écrit, la lecture et l'écriture : théories et didactiques. Paris : L'Harmattan.

Contreras, G. y Zúñiga, C. (2018). Concepciones sobre concepciones sobre retroalimentación del aprendizaje: Evidencias desde la Evaluación docente en Chile. Revista Actualidades Investigativas en Educación, 18(3), 1-25. Recuperado de https://www.scielo.sa.cr/pdf/aie/v18n3/1409-4703-aie-18-03-415.pdf

Crooks, T. (1988). The Impact of Classroom Evaluation on Students. Review of Educational Research, 58, 438-481. Recuperado de: http://www.jstor.org/stable/1170281

Cuq, J.-P. y Gruca, I. (2005). Cours de didactique du français langue étrangère et seconde. Paris: CLÉ Internacional.

Culham, R. (2015). Call a meeting with your writing teacher self. The reading teacher, 69, 219-222. doi: 10.1002/ trtr. 1383

Cuxim, M.M. (2018). Efectos de la retroalimentación correctiva en la producción escrita de estudiantes de inglés como lengua extranjera. (Tesis de Maestría). Universidad de Quintana Roo, México.

Desgagné, S. (1997). Le concept de recherche collaborative : l'idée d'un rapprochement entre chercheurs universitaires et praticiens enseignants. Revue des sciences de l'éducation, 23(2), 371 - 393. doi: https://doi.org/10.7202/031 $921 \mathrm{ar}$

Desgagné, S., Bednarz, N., Lebuis, P., Poirier, L. y Couture, C. (2001). L'approche collaborative de recherche en éducation : un rapport nouveau à établir entre recherche et formation. Revue des sciences de l'éducation, 27(1), 33-64. doi: https://doi.org/10.7202/012754ar

Domínguez-Gaona, R.; Crhová, J. y Molina-Landeros, C. (2015). La investigación colaborativa: las creencias de los docentes de lenguas. Revista de Iberoamericana de Educación Superior, 6(17), 120 - 134. Recuperado de https: //www.redalyc.org/pdf/2991/299141540007.pdf 
Duijnhouwer, H., Prins, F.J. y Stokking, K.M. (2012). Feedback providing improvement strategies and reflection on feedback use: Effects on students' writing motivation, process, and performance. Learning and Instruction, 22, 171-184. doi: https://doi.org/10.1016/j.learninstruc.2011.10.003

Durand, M.-J. y Poirier, S. (2012). La recherche collaborative au service du développement professionnel en ligne. Éducation et francophonie, 40(1), 119 - 137. doi: https://doi.org/10.7202/1010149ar

Eslami, E. (2014). The Effects of Direct and Indirect Corrective Feedback Techniques on EFL Students' Writing. Procedia - Social and Behavioral Sciences, 98, 445-452. doi: https://doi.org/10.1016/j.sbspro.2014.03.438

Evans, C. (2013). Making sense of assessment feedback in higher education. Review of educational research, 83, 70-120. doi: https://doi.org/10.3102/0034654312474350

Ferris, D. (1999). The case of grammar correction in L2 writing classes: a response to Truscott (1996). Journal of second language writing, 8(1), 1-11. doi: https://doi.org/10.1016/S1060-3743(99)80110-6

Ferris, D. y Roberts, B. (2001). Error feedback in L2 writing classes: how explicit does it to be?. Journal of second language writing, 10, 161-184. doi: https://doi.org/10.1016/S1060-3743(01)00039-X

Fithriani, R. (2019). ZPD and the benefits of written feedback in L2 writing: Focusing on students' perceptions. The reading matrix, 19 (1), 63-73. Recuperado de http://www.readingmatrix.com/files/20-c6t93b93.pdf

Fortin, M.-F. (2010). Fondements et étapes du processus de recherche : méthodes quantitatives et qualitatives. Québec : Chenelière éducation.

García, A. (2013). Estudio de percepción de universitarios alemanes sobre estrategias de retroalimentación correctiva de la expresión escrita. Revista de didáctica español como lengua extranjera, 17, 1-31. Recuperado de https://ww w.redalyc.org/articulo.oa?id=92152425009

Germain, C. (1993). Évolution de l'enseignement des langues: 5000 ans d'histoire. Paris : Clé international.

Guénette, D. (2010). La rétroaction corrective à l'écrit : pratiques et croyances, deux réalités parallèles?. La revue canadienne des langues vivantes, 66, 935 - 966. doi: https://doi.org/10.3138/cmlr.66.6.935

Halté, J.F. (1984). L'annotation des copies, variété ou base du dialogue pédagogique. Pratiques, 44, 61-69. Recuperado de https://www.persee.fr/doc/prati_0338-2389_1984_num_44_1_2463

Hattie, J. y Timperley, H. (2007). The Power of Feedback. Review of Educational Research, 77, 81 - 112. doi: https: //doi.org/10.3102/003465430298487

Hayes, J.R. y Flower, L.S. (1980). Identifying the organization of writing processes. En L.W. Gregg y E.R. Steinberg (dir.), Cognitive processes in writing (pp. 3-30). New Jersey: LEA.

Hyland, F. y Hyland, K. (2001). Sugaring the pill: praise and criticism in written feedback. Journal of second language writing, 10, 185-212. Recuperado de https://www.academia.edu/9592591/Sugaring_the_pill_praise_and_crit icism_in_written_feedback

Hyland, K. y Hyland, F. (2006). Feedback on second language students' writing. Language Teaching, 39, 83-101. doi: https://doi.org/10.1017/S0261444806003399

Jamalinesari, A. ; Rahimi, F. ; Gowhary, H. y Azizifar, A. (2015). The Effects of Teacher-Written Direct vs. Indirect Feedback on Students' Writing. Procedia-Social and Behavioral Sciences, 192, 116-123. doi: https://doi.org/10 $.1016 /$ j.sbspro.2015.06.018

Kluger, A.N. y DeNisi, A. (1996). The effects of feedback interventions on performance: a historical review, a metaanalysis, and a preliminary feedback intervention theory. Psichological bulletin, 119(2), 254-284. Recuperado de https://pdfs.semanticscholar.org/97cc/e81ca813ed757e1e76c0023865c7dbdc7308.pdf

Larsen-Freeman, D. y Anderson, M. (2016). Techniques and principles in language teaching. Oxford: Oxford University Press.

Lapointe, J. (2011). Étude des annotations d'un enseignant à la suite de l'enseignement explicite des stratégies de résolution de problemes mathématiques. (Tesis de Maestría). Universidad de Montreal, Canadá.

Leboeuf, P. (1999). “Très bien, Pascal, continue comme ça!" ou la lecture de l'explication dans le commentaire. Québec français, 115, 39 - 42. Recuperado de https://www.erudit.org/fr/revues/qf/1999-n115-qf1199433/56150ac/ 
Lee, I. (2007). Assessment for learning: Integrating Assessment, Teaching, and Learning in the ESL/EFL Writing Classroom. The Canadian Modern Language Review, 64, 199 - 213. doi: https://doi.org/10.1353/cml.2008.0 018

Lee, I. (2019). Teacher written corrective feedback: Less is more. Language Teaching, 52, 524-536. doi: 10.1017/ S0261444819000247

Lussier, D. y Turner, C. (1995). L'évaluation en didactique des langues. Quebec : Centre éducatif et culturel.

Mak, W.W.P. (2014). Implementing Innovative Feedback in L2 Writing: A Sociocultural Perspective. (Tesis de doctorado). Chinese University of Hong Kong, Hong Kong.

Manchón, R.M. (2011). Writing to learn the language: Issues in theory and research. En R.M., Manchón (dir.), Learning-to-write and writing-to-learn in an additional language (pp. 61-82). Amsterdam: John Benjamins.

Manchón, R.M.; Roca de Larios, J. y Murphy, L. (2000). La influencia de la variable "grado de dominio de la L2" en los procesos de composición en lengua extranjera: hallazgos recientes de la investigación. En C. Muñoz (dir.), Segundas lenguas: adquisición en el aula (pp. 277-297). Barcelona: Ariel lingüística.

Miedzo, M. y Chipo, M. (2019). The role of written feedback in numeracy in the primary school classroom. International Journal of Education, 11(2), 52-67. doi:https://doi.org/10.5296/ije.v11i2.14550

Min, T. (2013). A case study of an EFL writing teacher's belief and practice about written feedback. System, 41, 625-638. doi : https://doi.org/10.1016/j.system.2013.07.018

Morrissette, J. (1999). Pour une pratique du commentaire en trois temps. Québec français, 115, 52-54. Recuperado de: https://www.erudit.org/fr/revues/qf/1999-n115-qf1199433/56154ac/

Morrissette, J. (2013). Recherche-action et recherche collaborative: quel rapport aux savoirs et à la production de savoirs ?. Nouvelles pratiques sociales, 25(2), 35 - 49. doi: https://doi.org/10.7202/1020820ar

Nicol, D.J. y Macfarlane-Dick, D. (2006). Formative assessment and self-regulated learning: a model and seven principles of good feedback practice. Studies in Higher Education, 31, 199-218. doi: https://doi.org/10.1080/ 03075070600572090

Rakedson, T. y Baram-Tsabari, A. (2016). Assessing and improving L2 graduate students' popular science and academic writing in an academic writing course. Educational psychology, 10, 1-20. doi: https://doi.org/10.108 0/01443410.2016.1192108

Ramaprasad, A. (1983). On the definition of feedback. Behavioral Science, 28, 4-13. doi: https://doi.org/10.1002/b s.3830280103

Roberge, J. (2008). Rendre plus efficace la correction des rédactions : rapport PAREA. Montréal : Cégep AndréLaurendeau.

Robert, J.-P. (2002). Dictionnaire pratique de didactique du FLE. Paris : Ophrys.

Rodet, J. (2000). La rétroaction, support d'apprentissage?. Revue du conseil québécois de la formation à distances, 4, 45 - 73. Recuperado de https://edutice.archives-ouvertes.fr/edutice-00000482/document

Rouleau-Girad, E. (2015). La retroaction corrective écrite en francisation au Québec: Les techniques, les types d'erreurs et la compétence des apprenants à l'écrit (Tesis de Maestría). Universidad de Montreal, Canadá.

Sadler, D. R. (1989). Formative assessment and the design of instructional systems. Instructional Science, 18, 119-144.

Scallon, G. (2008). Évaluation formative et psychologie cognitive : mouvances et tendances. En J. Grégoire (dir.), Évaluer les apprentissages (pp. 158 - 173). Bruselas, Bélgica : De Boeck Supérieur.

Sheen, Y. (2011). Corrective Feedback, Individual Differences and Second Language Learning. New York: Springer.

Simard, C. (1999). L'annotation des textes d'élèves. Québec français, 115, 32 - 38. Recuperado de https://www.erud it.org/fr/revues/qf/1999-n115-qf1 199433/56149ac.pdf

Sow, M. (2015). Documenter les pratiques évaluatives de trois enseignants du primaire au Sénégal au regard de la rétroaction écrite en correction de textes d'élèves du CM 1. (Tesis de Maestría). Universidad de Montreal, Canadá.

Storch, N. y Tapper, J. (1997). Student annotations: what NNS and NS university students say about their own writing. Journal of second language writing, 6, 245-264. doi: https://doi.org/10.1016/j.jslw.2009.02.003 
Valdivia, S. (2014). Retroalimentación efectiva en la enseñanza universitaria. Blanco y negro, 5, 20-24. Recuperado de http://revistas.pucp.edu.pe/index.php/enblancoynegro/article/view/11388

Van der Maren, J.-M. (1996). Méthodes de recherche pour l'éducation. Bruselas : De Boeck.

Voelkel, S.; Varga-Atkins, T. y Mello, L. (2019). Students tell us what good written feedback looks like. FEBS Press, 10, 692-706. doi: https://doi.org/10.1002/2211-5463.12841

Vollmeyer, R. y Rheinberg, F. (2005). A surprising effect of feedback on learning. Learning and Instruction, 15, 589-602. doi: https://doi.org/10.1016/j.learninstruc.2005.08.001

Wiggins, G. (1998). Educative assessment: Designing Assessments to Inform and Improve Student Performance. San Francisco: Jossey-Bass.

\section{Anexo}

Definición de las siglas utilizadas en las figuras

Definición de las siglas utilizadas en las figuras

$\begin{array}{ll}\text { Siglas } & \text { Definición } \\ \text { AFFECPOSINF } & \text { Afectivo positivo informativo } \\ \text { AFFECNEGINF } & \text { Afectivo negativo informativo } \\ \text { METACONSSUG } & \text { Metacognitivo constructivo sugestivo } \\ \text { METACONSINF } & \text { Metacognitivo constructivo informativo } \\ \text { METAPOSINF } & \text { Metacognitivo positivo informativo } \\ \text { METAPOSSUG } & \text { Metacognitivo positivo sugestivo } \\ \text { METANEGSUG } & \text { Metacognitivo negativo sugestivo } \\ \text { METANEGINF } & \text { Metacognitivo negativo informativo } \\ \text { METHCONSSUG } & \text { Metodológico constructivo sugestivo } \\ \text { METHCONSINF } & \text { Metodológico constructivo informativo } \\ \text { METHCONSPRES } & \text { Metodológico constructivo prescriptivo } \\ \text { METHNEGINF } & \text { Metodológico negativo informativo } \\ \text { METHNEGPRES } & \text { Metodológico negativo prescriptivo } \\ \text { METHPOSSUG } & \text { Metodológico positivo sugestivo } \\ \text { METHPOSINF } & \text { Metodológico positivo informativo } \\ \text { METHPOSPRES } & \text { Metodológico positivo prescriptivo } \\ \text { COGNCONSINF } & \text { Cognitivo constructivo informativo } \\ \text { COGNCONSPRES } & \text { Cognitivo constructivo prescriptivo } \\ \text { COGNCONSSUG } & \text { Cognitivo constructivo sugestivo } \\ \text { COGNNEGINF } & \text { Cognitivo negativo informativo } \\ \text { COGNNEGSUG } & \text { Cognitivo negativo sugestivo } \\ \text { COGNNEGPRES } & \text { Cognitivo negativo prescriptivo } \\ \text { COGNPOSSUG } & \text { Cognitivo positivo sugestivo } \\ \text { COGNPOSINF } & \text { Cognitivo positivo informativo } \\ \text { COGNPOSPRES } & \text { Cognitivo positivo prescriptivo }\end{array}$

\section{INFORMACIÓN ADICIONAL}

Cómo citar: Cómo citar: Chao-Chao, K.W. y Durand, M.-J. (2021). La retroalimentación escrita en la producción escrita del francés L2. Revista Educación, 45(2). Recuperado de http://doi.org/10.15517/reve du.v45i1.43191 